\title{
La sinergia entre el diseño estructural y la dinámica de fluidos: el caso particular del velódromo de Medellín, en Colombia
}

\section{The synergy between structural design and fluid dynamics: the case of the cycle track of Medellin, in Colombia}

\author{
C. Muñoz Blanc $^{(*)}$
}

\section{RESUMEN}

En el presente trabajo se presentan las ventajas que la determinación prestacional de las acciones eólicas a partir de los métodos basados en la dinámica computacional de fluidos tiene en el diseño estructural de edificaciones de geometría ahusada y/o compleja como la que aquí se expone. El análisis del comportamiento del flujo eólico en diseños arquitectónicos y estructurales cada vez más etéreos y ligeros mediante avanzadas herramientas de simulación, las cuales utilizan una amplia fundamentación teórica basada en la dinámica de fluidos y en los medios continuos, ha permitido minimizar el impacto de las cargas eólicas en la dimensión de los elementos estructurales principales. Se muestran las metodologías de trabajo que han permitido optimizar la estructura principal del nuevo Velódromo de Medellín, en Colombia.

Palabras clave: Aerodinámica civil; dinámica de fluidos; análisis estructural; cargas eólicas; fuerzas de arrastre y sustentación.

\section{ABSTRACT}

This paper presents the advantages that the performance determination of wind actions from methods based in computational fluid dynamics has in the structural design of buildings with tapered or complex geometry. The analysis of the wind flow behaviour in lighter and more ethereal architectural and structural designs by means of advanced simulation tools, that use a wide theoretical ground based in fluid dynamics and continuum, has made possible to minimise the impact of wind loads in the size of the main structural elements. This paper shows the work methodologies that have allowed to optimize the main structure of the new Cycle track of Medellin, in Colombia.

Keywords: Aerodynamic analysis; fluid dynamics; structural design; wind loads; drag and lift forces.

(*) Dr. Arquitecto

Persona de contacto/Corresponding author: carlos.munoz@coac.net (C. Muñoz Blanc)

Cómo citar este artículo/Citation: Muñoz Blanc, C. (2016). La sinergia entre el diseño estructural y la dinámica de fluidos: el caso particular del velódromo de Medellín, en Colombia. Informes de la Construcción, 68(542): e146, doi: http://dx.doi.org/10.3989/ic.15.059.

Licencia / License: Salvo indicación contraria, todos los contenidos de la edición electrónica de Informes de la Construcción se distribuyen bajo una licencia de uso y distribución Creative Commons Attribution License (CC BY) Spain 3.o. 


\section{INTRODUCCIÓN}

El nuevo Velódromo de Medellín, en Colombia, diseñado por el equipo Mazzanti Arquitectos entre 2012 y 2014 (Figura 1), tiene como objetivo principal entregar a los ciudadanos una infraestructura deportiva de alto rendimiento, así como un espacio público, consistente en una base que resuelve los accesos y el contenido programático, y una cubierta que garantiza la integración paisajística y programática del edificio dentro del parque.

La cubierta del complejo, la cual abarca un área de aproximadamente $15.000 \mathrm{~m}^{2}$, se resuelve mediante unas estructuras tensadas de ruedas de bicicleta dispuestas en horizontal, siguiendo los esquemas planteados por Le Ricolais o la viga en forma de rueda de acero con barras tensadas, situada en el sótano de la casa Milà, de Antoni Gaudí (1). Se trata de siete ruedas principales, que cubren la totalidad de la superficie del Velódromo y sus gradas, de las cuales dos no tienen un soporte directo al suelo y se sustentan por las ruedas contiguas y mediante otras auxiliares que mantienen el equilibrio del sistema. La estructura principal de esta cubierta tensada está diseñada por BAC Engineering Consultancy Group.

Este diseño integral de la estructura de la cubierta implica la evaluación precisa de las cargas eólicas particularizadas, tanto para valorar las acciones reales que afectan a la estructura, como para garantizar la integridad de la membrana envolvente. Sin embargo, la compleja e inusual geometría de las ruedas que configuran el Velódromo propicia que se puedan crear efectos de presión y succión difícilmente evaluables a partir de los métodos analíticos recogidos en la normativa colombiana NSR-10 (2), tanto en la membrana exterior como en los espacios interiores de cada una de las ruedas de la cubierta. En el momento de desarrollar el estudio de Aerodinámica Civil toda la geometría del proyecto está definida por el equipo de diseño, tanto en la posición y el tamaño de las diferentes ruedas como en su proporción.
El comportamiento que un fluido tiene en su paso a través de un objeto sólido viene condicionado por lo que denominamos la aerodinámica del objeto sólido. La totalidad de las normativas vigentes (eurocódigos, normas americanas, etc.) determinan las acciones eólicas para aquellas formas más elementales, que de otra forma son las más habituales en Arquitectura.

Las últimas actualizaciones que se han hecho de todas las normas anteriormente indicadas ya reflejan las singularidades de flujo que se genera en las zonas de discontinuidad, como pueden ser las aristas y las esquinas del edificio, por ejemplo. En estas zonas de discontinuidad la intensidad del viento se ve incrementada de manera importante, como consecuencia directa de diversos factores como son las turbulencias y la separación de la capa límite (3). Si bien es cierto que en las geometrías elementales esta intensificación de la acción eólica queda perfectamente caracterizada, en una geometría tan compleja y de marcado carácter aerodinámico como es la cubierta que nos atañe, estas singularidades mostradas únicamente tienen la intención de reflejar un problema que necesita ser evaluado mediante modernas herramientas de análisis capaces de estudiar la aerodinámica particular.

El estudio combinado de cualquier sistema estructural con la aerodinámica del flujo eólico tiene sus orígenes en el campo de la aeronáutica, donde la interacción que se produce entre los elementos estructurales elásticos y la dinámica de fluidos da origen a un nuevo ámbito de conocimiento denominado aeroelasticidad. Este campo de la ciencia, desarrollado en gran medida por el científico, ingeniero y matemático Theodore Von Karman a partir de 1940 (4), toma una gran relevancia en el campo de la ingeniería aeroespacial durante el último cuarto del siglo xx, tras más de 70 años de avance en el campo de la aeronáutica. Se produce una rápida evolución desde el primer túnel de viento, diseñado de manera rudimentaria durante los primeros años del siglo xvIII, hasta los modernos túneles en los que se simulan condiciones hipersó-

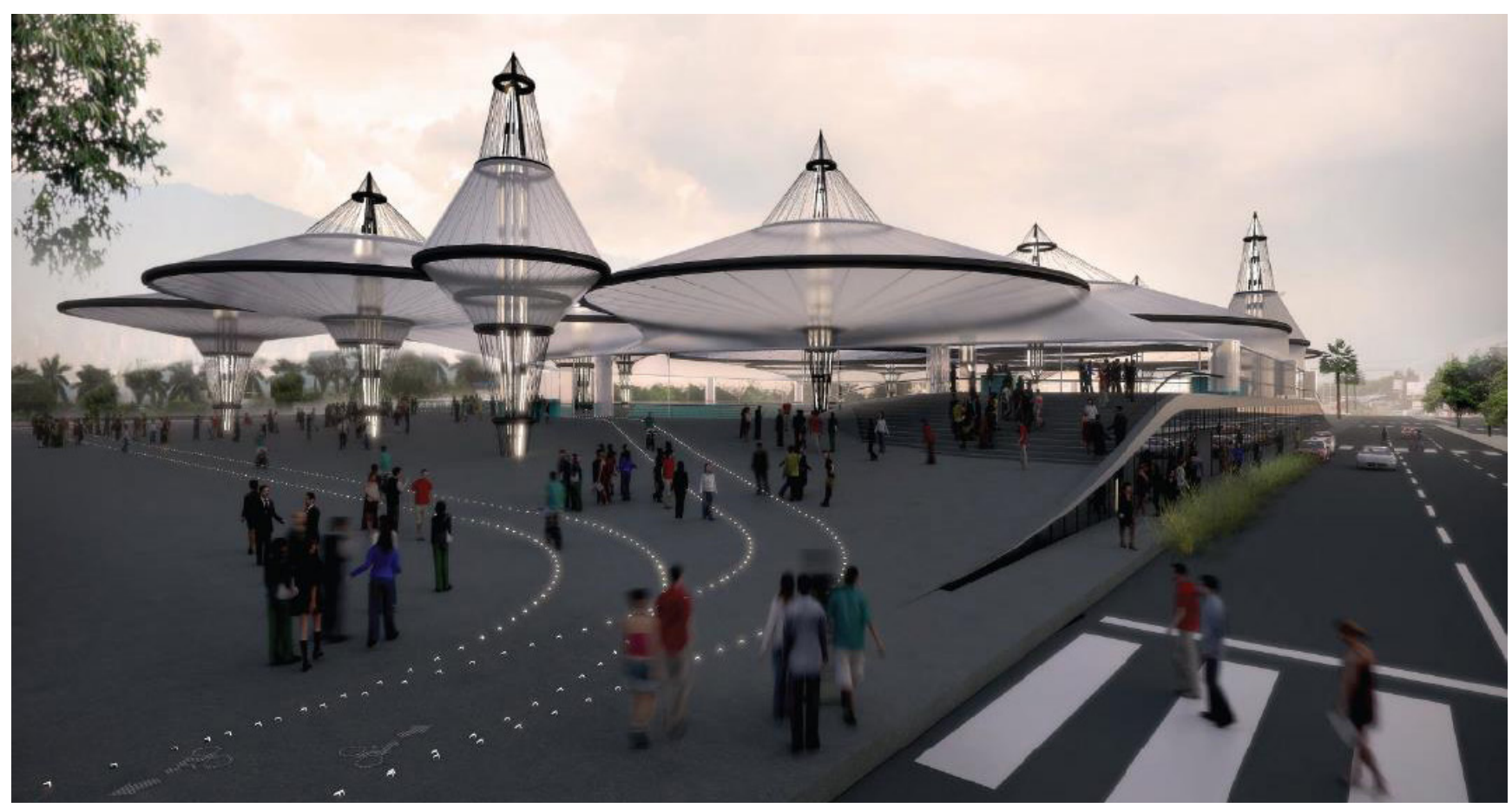

Figura 1. Fotomontaje de las «ruedas» que conforman la cubierta. Autor: Equipo Mazzanti Arquitectos. 
nicas extremas y los complejos análisis aeroelásticos que los complementan.

Sin embargo, en el ámbito de la Arquitectura, es a partir de las nuevas necesidades que se derivan de unas geometrías cada vez más complejas y esbeltas que surgen en los últimos tiempos, cuando se comienzan a desarrollar herramientas que permiten adaptar las tecnologías aeronáuticas al campo de la construcción. Lo que inicialmente se restringe al análisis de edificaciones complejas en túneles de viento experimentales se ha visto implementado durante la última década a las simulaciones computacionales y al estudio del comportamiento combinado entre el diseño estructural y la dinámica de fluidos.

Todas estas consideraciones plantearon en el momento de determinar las acciones eólicas sobre la cubierta un conjunto de nuevas exigencias en el proyecto que obligó a utilizar todos los recursos teóricos disponibles. El vertiginoso desarrollo de la Mecánica de Fluidos (5), junto con el nacimiento del método de los métodos finitos y la vertiginosa evolución de los ordenadores (6), permite hoy en día reproducir con una aproximación muy significativa el comportamiento de cualquier fluido alrededor de los cuerpos sólidos, considerando fenómenos tan complejos como la turbulencia o la viscosidad del fluido en la capa límite, evaluando cualquier geometría, por complicada que ésta sea.

\section{METODOLOGÍA DE ANÁLISIS}

\subsection{Generación del modelo}

El modelado inicial de la cubierta se realiza mediante programas Computer Aided Design (CAD) que permiten la parametrización de todos aquellos valores que definen la geometría de las ruedas; se realiza mediante el programa Revit Autodesk. Todo este trabajo inicial, tanto en CAD como con metodologías Building Information Modeling, permite que la gestión de la información del modelo con los programas de análisis estructural y de dinámica de fluidos sea mucho más ágil y eficaz. Este flujo de información se consigue gracias al desarrollo de los modelos de verificación estructural con Robot Autodesk y un programa propio de elementos finitos desarrollados en el departamento de I+D de BAC que permite la interpretación automática de los resultados de presiones del flujo obtenidas en Ansys FLUENT, v14. La geometría, generada inicialmente con el software Rhinoceros, es introducida en FLUENT mediante el programa DesignModeler (Ansys Workbench).

Un aspecto que tiene una destacada relevancia en la simulación computacional de un fenómeno físico cualesquiera es la definición del ámbito del dominio. Considerando las dimensiones de la cubierta del velódromo, $150 \times 100 \mathrm{~m}$ en planta $\mathrm{y}$ $20 \mathrm{~m}$ en altura, y mediante los criterios de buena práctica en la definición de dominios de simulación basados en la mecánica de fluidos (Figura 2), las dimensiones globales alcanzaron los $1.900 \times 600 \times 215 \mathrm{~m}$, garantizando esto el correcto comportamiento del paso del flujo a través de las ruedas de la cubierta.

La discretización del modelo de dinámica de fluidos se realiza mediante una malla desestructurada de volúmenes tetraédricos en la zona más próxima a las ruedas (Figura 3), permitiendo esto la perfecta adaptación a la compleja forma de la cubierta. El límite del volumen de la malla desestructurada queda definido como la distancia entre dicho límite y el objeto sólido del modelo numérico, adimensionalizada respecto del tamaño característico de la capa límite (Figura 4). En el resto del dominio se define una malla estructurada a partir de elementos hexaédricos, que permite una mayor precisión y calidad en los resultados obtenidos con un número inferior de elementos. Esta mayor precisión de la malla hexaédrica se obtiene gracias a la marcada direccionalidad del flujo existente entre la entrada y la salida del túnel eólico.

Debido a la naturaleza del flujo que se produce en las ruedas de la cubierta y el modelo turbulento escogido para la caracterización de la física del problema es de suma importancia la caracterización de la capa límite. Para poder captar todos los fenómenos viscosos que se producen en esta zona se recurre a un mallado estructurado en una zona tangente a la membrana de las ruedas, con un primer elemento en contacto con la pared de dos milímetros de altura (Figura 5). Estimando que los prismas que componen esta región tienen una base inferior a los $200 \mathrm{~mm}$, la relación entre la altura y la base nunca excede la proporción 1:100. A partir del primer elemento de dos milímetros se genera una malla estructurada de 15 ni-
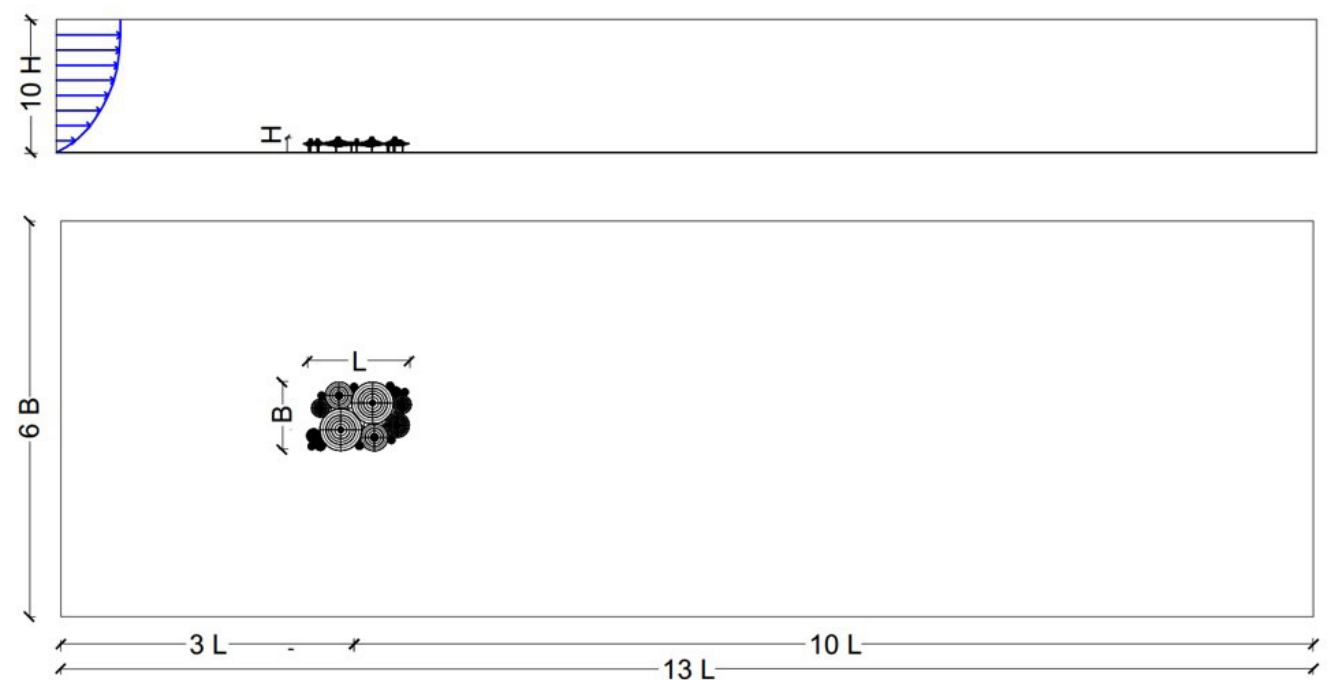

Figura 2. Dimensiones globales del dominio en relación al velódromo. 


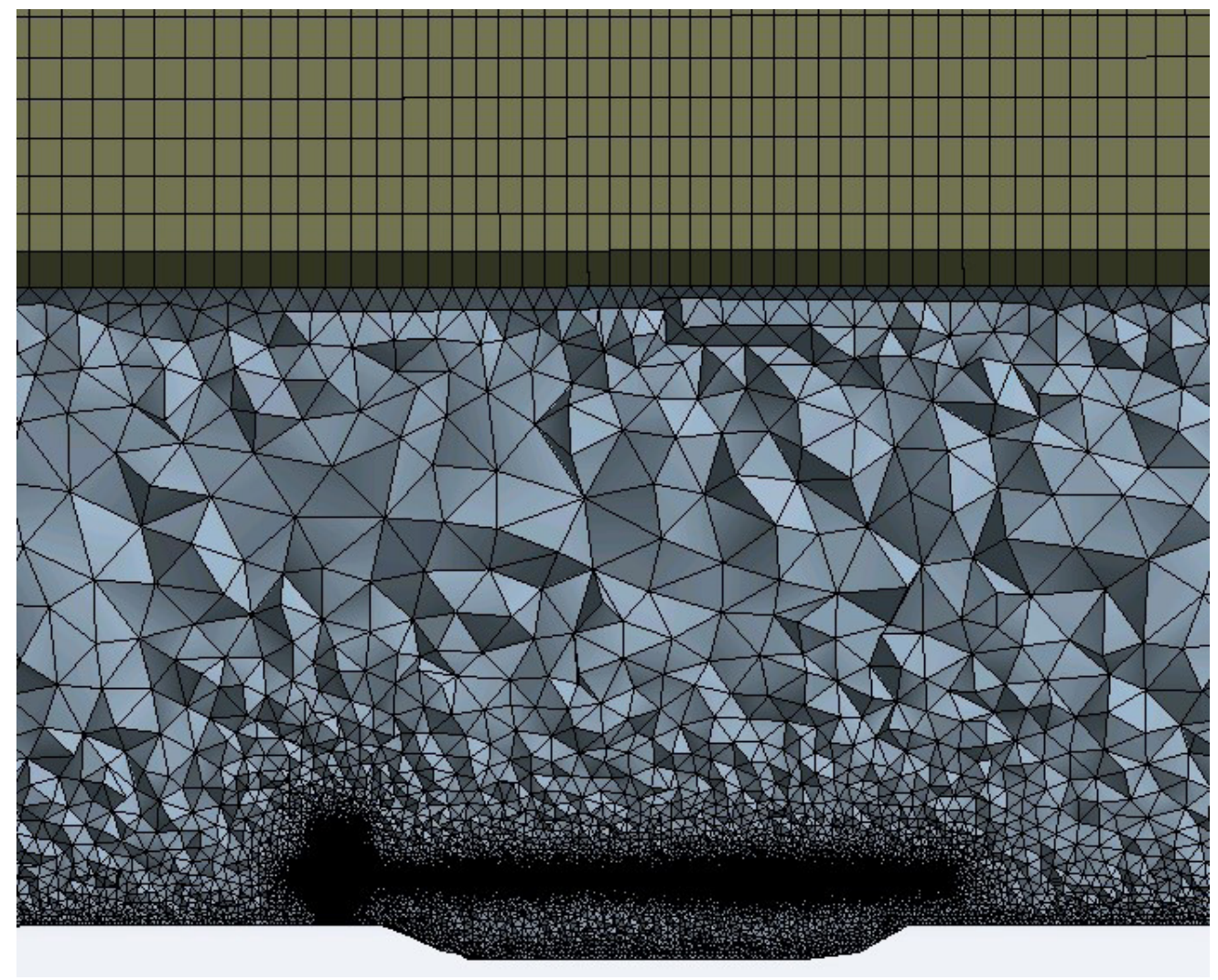

Figura 3. Discretización estructurada en la zona superior y desestructurada en las proximidades de las ruedas.

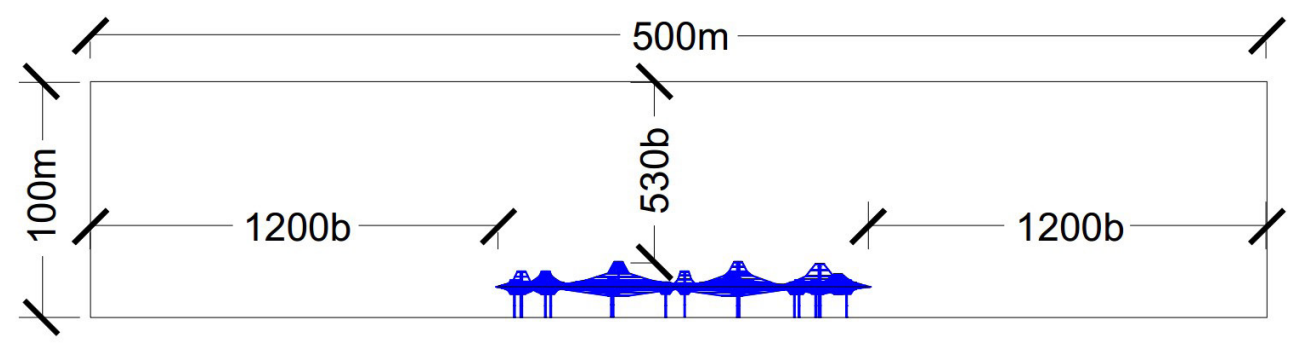

Figura 4. Límite de la malla desestructurada como la distancia hasta el cuerpo sólido adimensionalizada en relación al tamaño característico de la capa límite, siendo b la dimensión de la capa límite.

veles, produciéndose un incremento de espesor del $20 \%$ en cada uno de ellos respecto del anterior. Resulta así una malla estructurada de $144 \mathrm{~mm}$ para captar el gradiente de velocidad junto a las membranas de las ruedas.

\subsection{Parámetros de cálculo más significativos}

- Curva eólica

Una de las condiciones de contorno de mayor relevancia en el estudio es la definición de la curva eólica en la entrada del tunel de viento computacional. Según la norma colombiana se define ésta a partir de la ecuación [1], siendo sus variables los parámetros: $K_{z}$ el coeficiente de rugosidad en función de la altura, $K_{z t}$ el factor topográfico, $K_{d}$ el factor de direccionalidad, $V$ la velocidad del viento, e I el factor de importancia en función de la categoría del edificio.

$$
q_{z}=0,613 \cdot K_{z} \cdot K_{z t} \cdot K_{d} \cdot V^{2} \cdot I
$$

Para la determinación de la curva eólica se toma como referencia la velocidad básica, en la región IV del país, $33 \mathrm{~m} / \mathrm{s}$. Esta velocidad se toma, al igual que en la normativa americana y europea, a $10 \mathrm{~m}$ del suelo pero, a diferencia de los códigos europeos, para una ráfaga de tres segundos. Es determinante también el coeficiente de rugosidad en función de la altura, $K_{z}$, para una rugosidad en un entorno urbano con edificaciones de poca altura.

Para un periodo de retorno de 50 años el factor de importancia toma valor la unidad. En las proximidades del nuevo velódromo la topografía es horizontal, motivo por el que el factor topográfico también vale la unidad. Para independizar el estudio de aerodinámica civil de las combinaciones de acciones que se aplican en el diseño estructural el coeficiente direccional se toma como valor la unidad, estando esto del lado de la seguridad.

En un análisis únicamente mecánico de la estructura no sería preciso llevar a cabo un análisis dinámico de las ruedas; no 


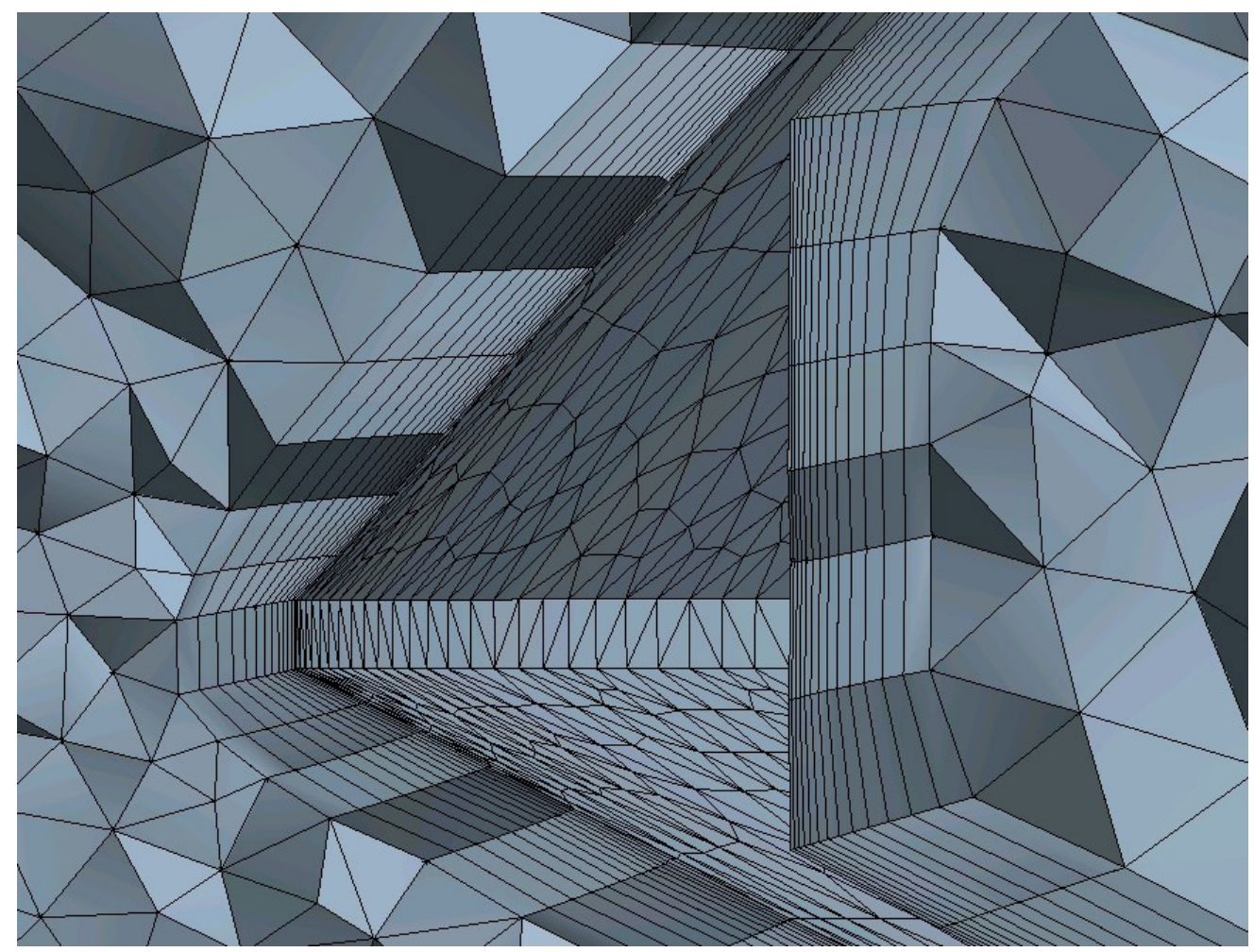

Figura 5. Malla estructurada con prismas en la zona de la capa límite junto a las membranas exteriores e interiores de las ruedas

existe un uso sobre la cubierta que haga necesario limitar la frecuencia natural de vibración de la estructura. Sin embargo, cuando se estudia la interacción entre el análisis estructural y la dinámica de fluidos es preciso analizar cuales son las frecuencias naturales de vibración. En este caso es determinante evaluar cual es la frecuencia en sentido vertical. En sentido horizontal la propia rigidez del sistema evita tener fenómenos aero-elásticos que puedan condicionar el diseño estructural.

En los bordes extremos del conjunto se pueden producir frecuencias inferiores a 1,0 Hz en sentido vertical. En este caso es preciso incorporar un factor de ráfaga, G [2], que amplifica la intensidad del viento en función de las propiedades dinámicas del sistema resistente.

La frecuencia natural de vibración en sentido vertical de una rueda aislada, hipótesis que se encuentra del lado de la seguridad, es de 0,56 Hz. Esta flexibilidad de las ruedas en sus extremos implica que el factor de ráfaga incremente la intensidad del viento en un $25 \%$ (Figura 6).

$$
P=q_{z} \cdot G \cdot C_{p}
$$

$P$ es la presión eólica que afecta a la estructura principal del edificio, la cual se calcula a partir de la presión básica obtenida en [1], el factor de ráfaga, $G$, y los correspondientes coeficientes de presión y succión $C_{p}$.

Se ha verificado el posible acoplamiento entre la frecuencia natural vertical de la estructura y la del desprendimiento de los vórtices. Las frecuencias de ambos fenómenos físicos han sido muy diferentes, motivo por el que no ha existido lock-in, es decir, acoplamiento entre ambas frecuencias. En caso contrario el desprendimiento de los vórtices ya no dependería de la velocidad del viento o de la geometría del elemento, sino que dependería de la vibración de la estructura.

Se ha estudiado también la generación de la calle turbulenta de Von Karman en la estela del velódromo; la propia geometría del velódromo y su proximidad al terreno eliminan esta posibilidad (Figura 7).

La distancia entre vórtices con rotación positiva y con rotación negativa es de aproximadamente 3,0 m (Figura 7). Considerando una velocidad del viento en la estela de $12 \mathrm{~m} / \mathrm{s}$, el tiempo existente entre las fuerzas alternas ascendentes y descendentes es de 0,25 s, es decir, una frecuencia de 4,0 Hz.

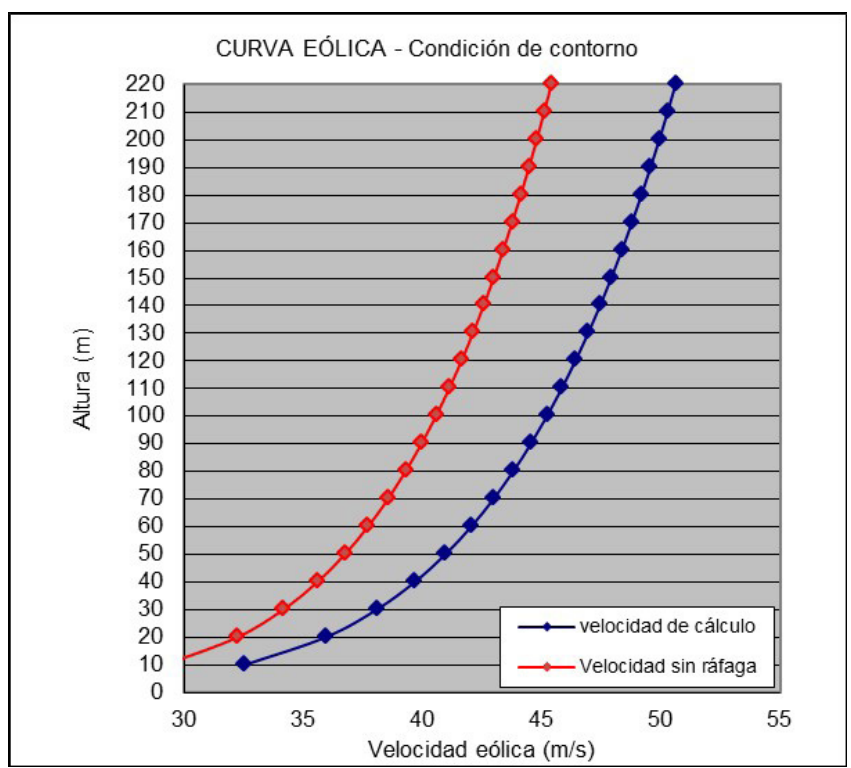

Figura 6. Comparación entre la curva eólica logarítmica sin incluir el factor ráfaga y la curva eólica de cálculo. 


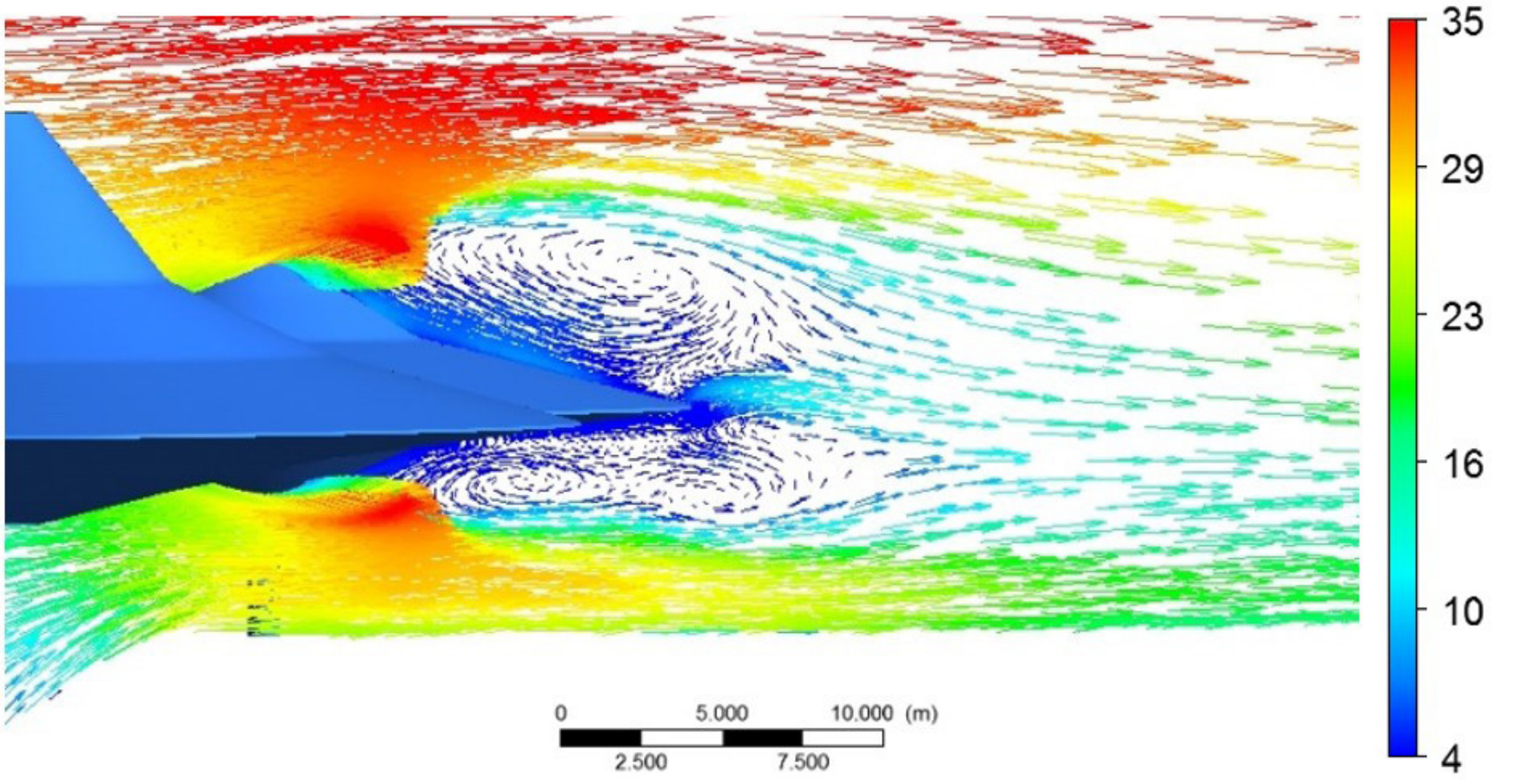

Figura 7. Vórtices generados en la estela del Velódromo.

\section{- Parámetros particularizados al emplazamiento}

Dada la altitud de la ciudad de Medellín sobre el nivel del mar (1.500 m.s.n.m.), se ha considerado una densidad del aire de $1,07 \mathrm{~kg} / \mathrm{m}^{3}$ en lugar de $1,225 \mathrm{~kg} / \mathrm{m}^{3}$, valor de referencia para la densidad del aire a nivel del mar, y una presión atmosférica de aproximadamente 85.000 Pa, en lugar de 101.325 Pa. La temperatura considerada es de $294 \mathrm{~K}$.

\section{- Tratamiento de las turbulencias}

El análisis que se presenta supone un reto a nivel de la dinámica del fluido y especialmente del tratamiento de la turbulencia. En la zona exterior de las ruedas lo más relevante es garantizar un correcto tratamiento de la capa límite, analizando zonas particulares en las que se produce la separación del flujo. Teniendo en cuenta el comportamiento del flujo en el exterior de las ruedas se podría recurrir a un tratamiento de la turbulencia basado en la resolución de dos ecuaciones, el método k- $\omega$ SST (7) (8) (9). Este método supone un avance respecto del tradicional método $\mathrm{k}-\varepsilon$, principalmente en la proximidad de los elementos sólidos, donde las funciones de pared adquieren mayor relevancia y donde la separación del flujo puede condicionar los resultados obtenidos.

Este método, k- $\omega$ SST, siempre ha mostrado una elevada precisión para la determinación de las presiones eólicas y el comportamiento del flujo en análisis aerodinámicos. Prueba de la precisión de este modelo es la incorporación del mismo en los test elaborados por el Instituto Americano de Aeronáutica y Astronáutica (A.I.A.A.) para la determinación de los coeficientes de presión en el $2 .^{\circ}$ seminario sobre predicción de fuerzas de presión el año 2003 (10). Los resultados experimentales obtenidos en el citado seminario en túneles de viento tienen una muy buena correlación con las simulaciones computacionales desarrolladas.
Sin embargo, es importante evaluar que sucede en el interior de las ruedas. Una vez analizadas las primeras simulaciones se observa el marcado carácter turbulento con flujo rotacional que se produce en el interior de las mismas. Para poder simular correctamente un flujo turbulento con una importante componente tangencial, y teniendo en cuenta que una misma simulación no permite la utilización de diferentes modelos de tratamiento de la turbulencia en diferentes ámbitos, se recurre finalmente a la resolución de los fenómenos turbulentos en todo el dominio computacional mediante el Método Reynolds-Stress de 7 ecuaciones (R.S.M.) (11). Este método, que incorpora todas las componentes tensionales del medio fluido, tanto normales como tangenciales, es mucho más preciso para la resolución de flujos rotacionales (12), pero tiene el inconveniente de implicar un coste computacional dos veces superior a los métodos basados en 2 ecuaciones.

\section{- Proceso de calibración del modelo}

En cualquier simulación computacional de mecánica de fluidos es básico la obtención de un modelo donde todos los parámetros de cálculo estén correctamente calibrados, y con una independencia clara entre los resultados obtenidos y la discretización espacial realizada.

Para lograrlo se desarrollan diversos modelos simplificados, estudiando una rueda aislada, en la que se analiza la capa límite y el flujo rotacional (Figura 8). Se determina así la dimensión mínima de los elementos discretos que permiten garantizar la independencia de los resultados, estudiando el comportamiento del flujo con diferentes tamaños de mallado, considerando que la dimensión es adecuada cuando variaciones en éste no comportan diferencias en los resultados obtenidos.

Para garantizar la calidad de las simulaciones resulta imprescindible el correcto tratamiento del flujo en las proximidades de las paredes, en la zona denominada subcapa viscosa 


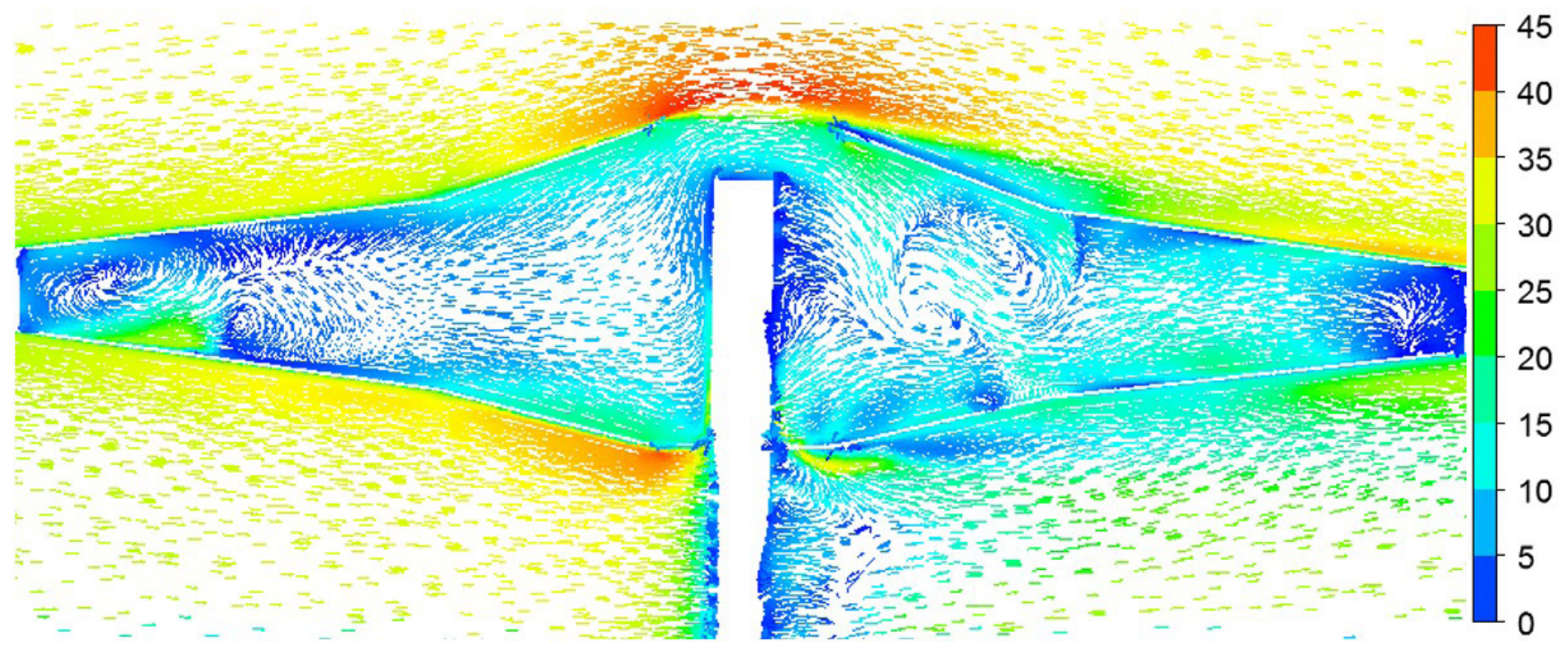

Figura 8. Vectores de velocidad en el interior de una rueda aislada.

(Figura 9), donde el gradiente de velocidad del flujo es muy marcado.

Existen dos estrategias para caracterizar correctamente el flujo en las proximidades de las paredes: la simulación numérica no simplificada de las turbulencias, mediante mallados extremadamente finos en las proximidades de las paredes, o bien mediante funciones de pared. Se recurre aquí a la incorporación de estas funciones de pared estándar de tipo logarítmico (13), conllevando esto una serie de suposiciones sobre el perfil de velocidades en la zona más próxima a la pared, para obtener así la tensión cortante. Debido a esto es preciso limitar el parámetro adimensional $\mathrm{Y}^{+}$[3] a valores comprendidos entre 30 y 300, situando así el primer nodo de cálculo exterior a la subcapa viscosa y garantizando que los resultados se adecuan a las suposiciones realizadas mediante las funciones de tipo logarítmico.

$$
Y^{+}=\frac{Y \cdot U_{t}}{v}
$$

Donde $Y$ es la distancia desde la superficie de la pared hasta el nodo de integración del primer volumen finito.

$$
U_{\tau}=\sqrt{\frac{\tau_{w}}{\rho}}
$$

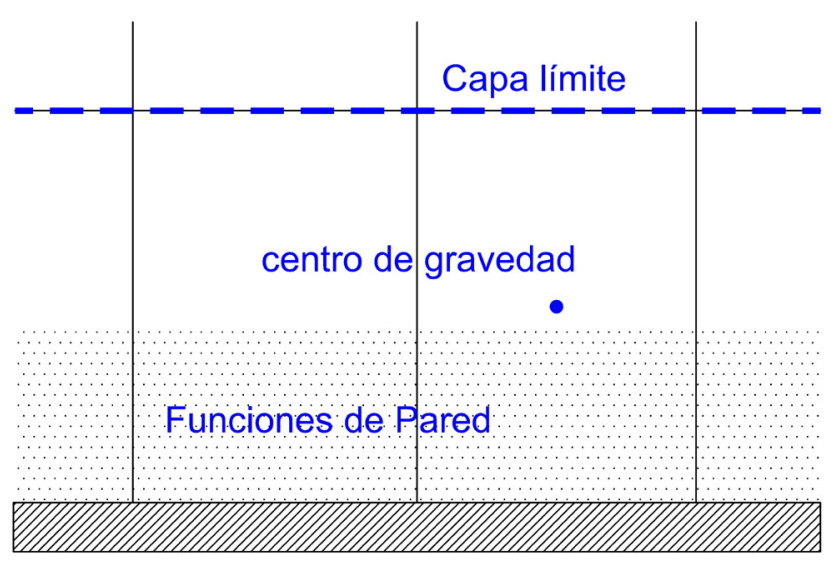

$$
\begin{gathered}
\tau_{w}=\frac{1}{2} \cdot C_{f} \cdot \rho \cdot U_{\infty}^{2} \\
C_{f}=0,058 \cdot R e_{l}^{-0,2}
\end{gathered}
$$

Se determina un valor de $C_{f}$ de o,042 para un número de Reynolds de 500.000 (flujo turbulento). El valor de definido según la ecuación [5], toma valor $2,91 \mathrm{~kg} /\left(\mathrm{m} \cdot \mathrm{s}^{2}\right)$. La velocidad tangencial a la pared según [4] es de $1,65 \mathrm{~m} / \mathrm{s}$, considerando una viscosidad del aire de $1,8 \cdot 10^{-5} \mathrm{~kg} /(\mathrm{m} \cdot \mathrm{s})$, con lo que para conseguir limitar el parámetro adimensional $Y^{+}$[3] a 200, el primer volumen finito debe tener una altura de dos milímetros.

\section{- Convergencia de los resultados}

Se han utilizado diversos parámetros para garantizar la correcta resolución del modelo. En primer lugar, se limita el valor del residual o desequilibrio en las ecuaciones de gobierno del fluido y de la turbulencia a $10^{-4}$. Los residuales son calculados en la solución final de cada una de las iteraciones realizadas para un flujo no transitorio, para cada una de las variables del modelo numérico.

Para determinar el valor del residual en cada una de las celdas del dominio, una vez realizada la discretización de cada

Figura 9. Esquema de la subcapa viscosa y de la zona turbulenta contenida en la capa límite. 
una de las ecuaciones de conservación de las variables, hay que evaluar el desequilibrio que se produce en la ecuación diferencial parcial de cada variable general en un volumen finito, dada de forma simplificada por la forma algebraica [7], habiéndose reordenado cada una de las ecuaciones diferenciales parciales de manera que el resultado para el volumen finito en estudio es una función de los valores que adquiere la variable en las celdas adyacentes, del campo de flujo y de la propia discretización del dominio.

$$
a_{p} \varnothing_{p}=\sum_{n b} a_{n b} \cdot \varnothing_{n b}+b
$$

Donde el término situado a la izquierda de la ecuación representa el valor de una variable dada en el volumen finito en estudio y la parte situada a la derecha de la ecuación representa en su primer término el sumatorio del valor de la variable en estudio para todos los volúmenes adyacentes al volumen en estudio y el segundo término es la parte constante del término fuente del campo de flujo para una condición de contorno dada. El subíndice $n b$ hace referencia a las celdas que son adyacentes al volumen finito referenciado.

En base a estas consideraciones se puede determinar que el valor residual para la totalidad del dominio queda definido de la siguiente manera [8]:

$$
R^{\varnothing}=\sum_{\text {Celdas } P}\left|\sum_{n b} a_{n b} \cdot \varnothing_{n b}+b-a_{P} \varnothing_{p}\right|
$$

Pero obtener un valor reducido de las residuales no es garantía suficiente para poder establecer que el modelo ha convergido correctamente. Los parámetros aerodinámicos de arrastre y sustentación también deben ser precisos. Se ha estudiado la variación de estos parámetros para cada iteración, monitorizando la variación de los valores de arrastre global y sustentación. Se ha verificado que no se producían variaciones significativas en el valor de estos dos parámetros de estudio.

Por último, se ha incluido la monitorización del desajuste entre el flujo de entrada y el de salida, estableciendo como criterio de convergencia un valor de $10^{-3}$. Este criterio implica la conservación de la masa del flujo en el dominio numérico. Debe existir equilibrio entre los flujos de entrada del dominio y los flujos de salida. La conservación de la masa garantiza que no se producen desequilibrios numéricos en las ecuaciones constitutivas del comportamiento del flujo. Un desequilibrio máximo del 0,1\% es garantía suficiente de la calidad en los resultados.

\section{- Estudio en 8 direcciones}

Se estudian 8 direcciones diferentes de incidencia del viento sobre la cubierta. Además de estudiar la acción eólica en las diferentes direcciones, también se realizan estudios de idoneidad del cerramiento perimetral del velódromo. Dada la condición de espacio deportivo donde se pretenden poder homologar records de velocidad, se plantea que la intensidad del viento en el interior no sea significativa. El proyecto de Arquitectura incorpora un cerramiento para que la acción del viento no sea determinante en la zona de la pista del velódromo. No obstante, teniendo en cuenta que este cerramiento puede ser un elemento efímero, todo el análisis estructural se realiza sin incluirlo, produciéndose unos resultados conservativos.

La Unión Ciclista Internacional (UCI), en su reglamento, no especifica ningún límite en el valor máximo del viento cuando se lleva a cabo la homologación de récords.

\section{RESULTADOS OBTENIDOS}

- Obtención de las fuerzas de presión y de arrastre reales en la cubierta

El análisis de la acción eólica en las 8 direcciones eólicas, permite obtener los mapas de presiones sobre la membrana que define la geometría de la cubierta, pudiendo observarse la hipótesis del viento incidiendo en sentido longitudinal (Figura 10). Se confirma con los mapas de presión que el interior de las ruedas se ve sometido a un estado de sub-presión. Esto implica que tanto para el diseño de la estructura principal realizado por BAC como para el de la membrana

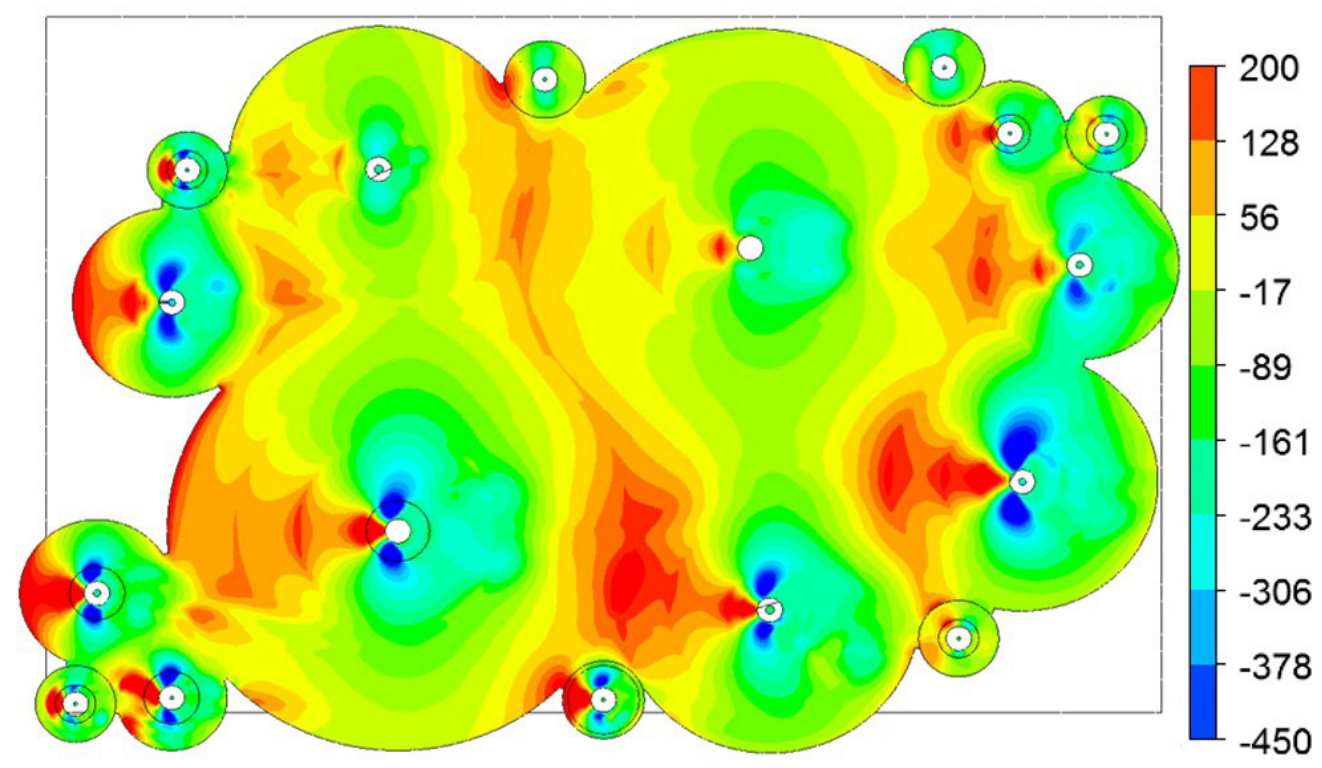

Figura 10. Presiones en el exterior de la membrana superior de la cubierta del Velódromo [Pa]. 
las fuerzas de presión/succión exteriores se vean compensadas a nivel global con las succiones producidas en el interior.

Este juego de presiones/succiones entre el exterior/interior, producido tanto en la membrana superior de las ruedas como en la membrana inferior, comporta el análisis de 4 superficies de $15.000 \mathrm{~m}^{2}$ para 8 direcciones eólicas y con variaciones en los datos de entrada. La interpretación de más de $500.000 \mathrm{~m}^{2}$ de presiones y la posterior aplicación en los modelos de estructuras puede llegar a ser un trabajo largo y tedioso, además de con una probabilidad de errores importantes si no se sistematiza. A esto se le debe añadir que en este caso no únicamente interesa analizar las presiones que se generan; una superficie expuesta tan elevada de membrana obliga a estudiar el efecto de las fuerzas de arrastre.

\section{- Tratamiento y evaluación de los resultados obtenidos}

Para poder sistematizar el análisis y la introducción de datos se desarrolla una rutina en lisp. Mediante Ansys FLUENT se obtiene un fichero de datos con las coordenadas de los puntos de la malla que define el contorno de las ruedas, a los que se asocia el valor de la presión y la fuerza de arrastre en las tres coordenadas cartesianas. Este fichero, con más de 500.00o coordenadas y sus respectivos resultados, es interpretado por el programa lisp sobre la plataforma de AutoCAD, logrando generar una nueva malla en la que se promedian los valores obtenidos en cada nodo de una parcela ortogonal de aproximadamente $1 \times 1 \mathrm{~m}^{2}$. Esta primera operación permite pasar de tener una malla desestructurada de nodos cada 100-200 $\mathrm{mm}$, con sus respectivos resultados, a una malla ordenada de precisión $1 \times 1 \mathrm{~m}^{2}$.

Posteriormente los datos son introducidos automáticamente mediante coordenadas en el programa de análisis estructural. Se muestra en la Tabla 1 los valores medios de presión/succión obtenidos para cada una de las membranas, en función de la dirección eólica. Hay que destacar que estos son valores medios. El estudio permitió detectar zonas de concentración de presión/succión donde de manera localizada se adoptaron las soluciones resistentes necesarias.

El estudio también se utilizó para el análisis de las acciones sobre las membranas. Esta acción se define como la suma del efecto eólico en el exterior y el interior de la rueda.

Tabla 1. Presión media del viento $\left(\mathrm{N} / \mathrm{m}^{2}\right)$ en función de la dirección eólica analizada.

\begin{tabular}{|l|c|c|}
\cline { 2 - 3 } \multicolumn{1}{c|}{} & \multicolumn{1}{c|}{$\begin{array}{c}\text { Exterior } \\
\text { Inferior } \\
\left(\mathbf{N} / \mathbf{m}^{2}\right)\end{array}$} & $\begin{array}{c}\text { Exterior } \\
\text { Superior } \\
\left(\mathbf{N} / \mathbf{m}^{2}\right)\end{array}$ \\
\hline Dirección Norte & $-8,2$ & $-33,0$ \\
\hline Dirección Este & 6,6 & $-41,0$ \\
\hline Dirección Sur & $-0,3$ & $-28,0$ \\
\hline Dirección Oeste & 9,0 & $-54,0$ \\
\hline Dirección Noreste & 6,8 & $-41,0$ \\
\hline Dirección Noroeste & 15,5 & $-34,0$ \\
\hline Dirección Sureste & 7,7 & $-33,0$ \\
\hline Dirección Suroeste & 6,9 & $-47,0$ \\
\hline
\end{tabular}

Cuando se llevan a cabo estudios de aerodinámica civil es interesante comparar los valores obtenidos en las simulaciones computacionales con los valores establecidos en la normativa vigente. En el caso del velódromo de Medellín, con una geometría compleja y espacios interiores sometidos a sub-presión, resultaba complicado poder establecer una comparativa realista.

Sin embargo, si se prescinde del estado de sub-presión generado en el interior, y se comparan los valores obtenidos únicamente en la membrana superior exterior, se pueden extraer las siguientes conclusiones:

La presión básica $q_{z}$ según la norma colombiana es de 0,556 $\mathrm{kN} / \mathrm{m}^{2}$ a $20 \mathrm{~m}$ de altura [1]. Considerando el factor de ráfaga [2] la presión básica es de $0,690 \mathrm{kN} / \mathrm{m}^{2}$.

Comparando este valor con los valores indicados en el CTE-A, para una velocidad básica medida para una ráfaga de 10 minutos de $26 \mathrm{~m} / \mathrm{s}$ (valor típico en el centro de la península Ibérica), y aplicando el coeficiente de exposición en zona urbana correspondiente a una altura de $20 \mathrm{~m}$, el cual incluye los efectos turbulentos medidos para una ráfaga de 3 segundos, el valor de la presión eólica sería de $0,966 \mathrm{kN} / \mathrm{m}^{2}$.

Más complicado es poder definir el coeficiente de presión externa en un edificio con la geometría de la cubierta externa del velódromo. Según los resultados obtenidos, la succión media máxima obtenida en las simulaciones es de $-54,0 \mathrm{~N} / \mathrm{m}^{2}$. Teniendo en cuenta la presión básica, el coeficiente de presión medio en la cubierta del velódromo es de -0,078. No obstante, un área significativa de la membrana se ve sometida a succiones de -100 Pa (Figura 10). Esto implica un coeficiente eólico de succión de -0,15, valor significativamente reducido. Este valor del coeficiente de presión es sensiblemente más reducido que si tomásemos como referencia diferentes figuras geométricas indicadas en la NSR-10, como por ejemplo una cubierta a dos aguas en diente de sierra, donde el valor sería de -1,4.

La componente horizontal de las acciones eólicas, considerando ésta como la integración de las presiones de arrastre que actúan sobre la membrana (Figura 11) y la fuerza descompuesta que actúa sobre cada uno de los planos inclinados de las ruedas, está comprendida entre 502 y $575 \mathrm{kN}$ dependiendo de la dirección del viento analizada.

La superficie proyectada perpendicular a la acción eólica depende de la dirección evaluada, variando entre los 835 y los $1.145 \mathrm{~m}^{2}$.

Esto implica que el coeficiente de arrastre, $C_{d}$, de la cubierta del velódromo está comprendido entre 0,87 y 1,04 según [9], siendo las variables que determinan el coeficiente la fuerza total, $F$, el área proyectada sobre la que incide la acción eólica, $A$, la densidad del aire, $\rho$, y la velocidad del aire, $v$. En base a los datos teóricos se establece que el coeficiente de arrastre, $C_{d}$, para un disco circular, es de $\sim 0,90-1,00$, para los números de Reynolds que registra el flujo que se produce alrededor del objeto (14).

$$
C_{d}=\frac{2 \cdot F}{A \cdot \rho \cdot v^{2}}
$$




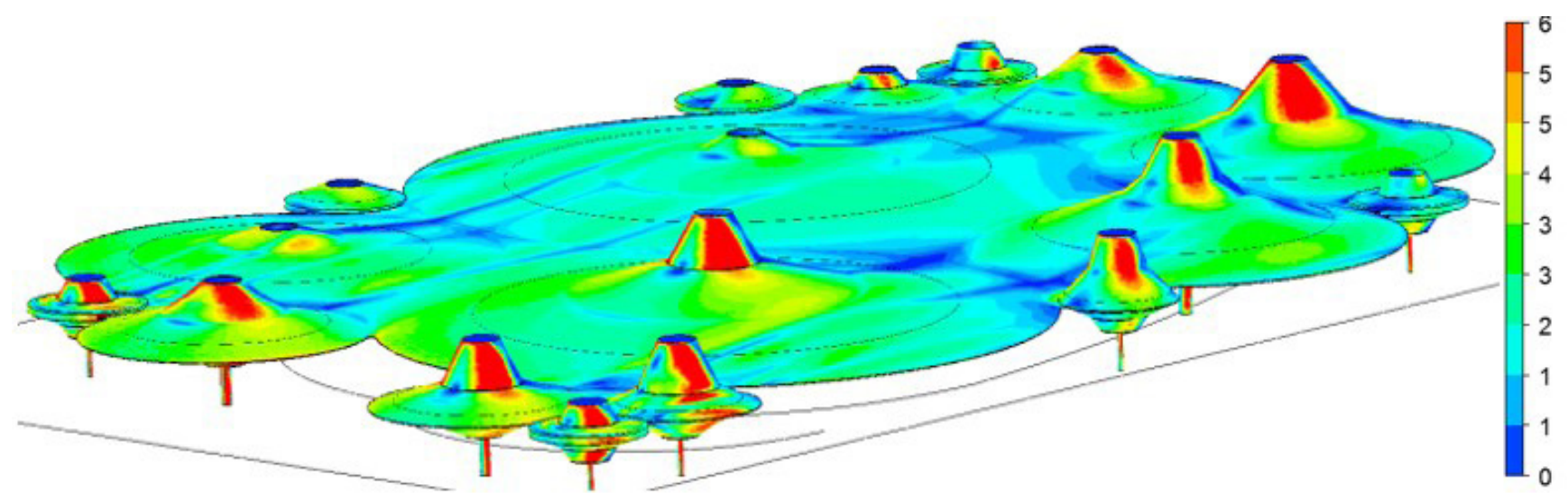

Figura 11. Presión que produce las fuerzas de arrastre en el exterior de la membrana superior de la cubierta del Velódromo [Pa].

Aunque las áreas proyectadas en cada una de las direcciones estudiadas difiere un $40 \%$, la fuerza de arrastre para cada una de las direcciones no varía más de un $12 \%$. Debido a la compleja geometría de la construcción, la influencia de dicha área vertical proyectada del objeto queda en un segundo plano debido a las siguientes consideraciones:

a) Existe una importante superficie que genera unas fuerzas tangenciales muy similares en cada una de las direcciones evaluadas. Estamos tratando un objeto aerodinámico.

b) La geometría de la cubierta, con un elevado factor aerodinámico, implica que la componente principal que genera las fuerzas de arrastre sea la horizontal, resultante de la descomposición de la presión sobre la membrana y las fuerzas de rozamiento, en vez de la proyección sobre el plano vertical de todo el elemento de cubierta.

\section{CONCLUSIONES}

El presente estudio muestra la necesidad de llevar a cabo estudios de Aerodinámica Civil cuando se desarrolla el diseño de geometrías complejas. En el caso del Velódromo de Medellín, el trabajo colaborativo entre los diferentes equipos ha permitido evaluar las cargas eólicas reales que afectan al edificio.

La geometría que define la cubierta queda fuera de cualquier objeto asimilable a los coeficientes de presión exterior que se incluyen en la normativa vigente. Además, la singularidad de la cubierta, con un espacio interior sometido a un estado de succión constante, hace imprescindible el desarrollo de estudios de aerodinámica civil basados en la dinámica de fluidos. Se ha podido ver como las fuerzas de presión/succión sobre la membrana tienen valores medios muy reducidos comparados con los valores prescriptivos, por la dificultad de aplicar coeficientes de presión adecuados a la geometría real. Se ha evaluado también la fuerza de arrastre que incide en la estructura principal del velódromo, garantizando que se incluyen aquí tanto la fuerza de presión como las fuerzas de arrastre sobre la membrana.

El proceso de simulación avanzada que aquí se ha presentado ha permitido optimizar el diseño de los elementos estructurales con un conocimiento preciso del comportamiento frente a las acciones eólicas de una cubierta de geometría extremadamente singular.

\section{AGRADECIMIENTOS}

Se quiere agradecer al equipo Mazzanti Arquitectos su predisposición desde el primer momento para realizar un trabajo de diseño en paralelo al equipo de ingeniería. También se quiere agradecer la autorización para la publicación de las imágenes que sirvieron para ganar el concurso público.

Igualmente, se quiere agradecer al equipo de diseño de la estructura de BAC Engineering Consultancy Group que realizó el diseño de la estructura el trabajo conjunto con el departamento de simulaciones avanzadas C.F.D.

\section{REFERENCIAS}

(1) Brufau i Niubó, R. (2006). La rueda de bicicleta como modelo estructural. Quaderns d'Arquitectura i Urbanisme, 250: 86-91.

(2) Reglamento Colombiano de Construcción Sismo Resistente. NSR-10. Título B - Cargas. Capítulo B.6 - Fuerzas de viento.

(3) Streeter, V., Wylie, B. (1988). Parte 1. Capítulo 6: Flujos externos. Mecánica de Fluidos, 8. a edición, pp. $247-261$. McGraw Hill.

(4) Garrick, L. E. (1976). Aeroelasticity-Frontiers and Beyond. 13. ${ }^{\text {a }}$ Conferencia Von Karman. Journal of Aircraft, 13(9): 641-657.

(5) Liñán, A. (2000). La mecánica de fluidos en los albores del siglo xx. Discurso inaugural del año académico $2000-2001$. Real Academia de Ciencias Exactas, Físicas y Naturales, 94(4): 535-552.

(6) Millán, G. (1978). La resolución numérica de los problemas aerodinámicos. Discurso inaugural del año académico 1978-1979. Real Academia de Ciencias Exactas, Físicas y Naturales.

(7) Samy, M., Mofreh, H. (2009). A Comparative Study of Turbulence Models Performance for Turbulent Flow in a Planar Asymmetric Diffuser. World Academy of Science, Engineering and Technology, 53. 
(8) Menter, F. R. (1994). Two-Equation Eddy-Viscosity turbulence Models for Engineering Applications. AIAA Journal, 32 : 1598-1605.

(9) Menter, F. R., Kuntz, M., Langtry, R. (2003). Ten Years of Industrial Experience with the SST turbulence Model. Turbulence, Heat and Mass Transfer 4.

(10) http://aiaa-dpw.larc.nasa.gov/Workshop2/workshop2.html (2003).

(11) Launcher, B. E., Spalding, D. B. (1974). The Numerical Computation of Turbulent Flows. Computer Methods in Applied Mechanics and Engineering, 3: 269-289.

(12) Balestrin, E., Luciano, R. D., Noriler, D., Decker, R. K., Meier, H. F. (2015). Sensibility Study of the Reynolds Stress Model Parameters for Swirling Flows in Cyclones. Chemical Engineering Transactions. AIDIC, 43: 1159-1164.

(13) Pope, S. B. (2000). Parte II: Modelling and Simulation, Cap. 11.7: Near Wall treatments. Turbulence flows, pp. $442-444$. Cambridge: Cambridge University Press.

(14) Sadraey, M. (2009). Cap. 3. Drag Force and Drag Coefficient. Aircraft Performance analysis. VDM Verlag Dr. Müller. 